\title{
LAS AFP EN PERÚ INCURRIERON EN EL USO DE PUBLICIDAD ENGAÑOSA Y DESINFORMACIÓN AL INICIAR SUS OPERACIONES, LO CUAL PRESUME DELITO DE ESTAFA
}

\author{
AFP IN PERU INGURRED USE MISLEADING ADVERTISING AND MISINFORMATION WHEN IT BEGAN \\ OPERATIONS, WHICH BOASTS SWINDLE \\ Juan Manuel Barreda Guerra* \\ jumabague@yahoo.es
}

[RECEPCIÓN: AGOSTO 2015 / CONFORMIDAD: NOVIEMBRE 2015]

\section{RESUMEN}

La investigación pretende demostrar que en la década de los 90 en nuestro país, tiempo en el que la Oficina de Normalización Previsional del Sistema Nacional de Pensiones (SNP) se encontraba una vez más en álgido momento económico y financiero, lo que originaba que la asignación económica por concepto de pensión de jubilación que recibían los pensionistas de aquel entonces, se encontraba lejos de poder atender y cubrir si quiera un $40 \%$ de la canasta familiar básica para subsistir. Sin embargo, en el año 1993, dentro de un gobierno de corte dictatorial e inconstitucional, se da inicio mediante Decreto Ley N ${ }^{\circ} 25897$ al nuevo Sistema Privado de Pensiones, sistema por el cual se crean las Administradoras de Fondos de Pensiones (AFP), entidades privadas cuya misión empresarial primigenia fue captar la mayor cantidad de jubilados que se desafiliaran del sistema estatal a fin de que se inscribieran y afilien en una determinada AFP para trasladar al inscrito junto con un bono de reconocimiento en soles otorgado por la ONP por sus aportaciones efectuadas. Estas aportaciones debían alcanzar las 48 cuotas consecutivas con las cuales podría evaluar alguno de los periodos establecidos para los años 1992, 1996 y 2001 para la tramitación de su bono de reconocimiento, monto pecuniario que el Estado otorgaría al nuevo afiliado inscrito en una AFP, con el propósito de que éste constituya un fondo de ahorro en su cuenta individual como aportante (trabajador ahorrista) en el nuevo sistema previsional. Esto se daba bajo el ofrecimiento que al trabajador que ahorrase por más de dos o tres décadas, el sistema le ofrecía un nivel de pensión de jubilación que le permitiría vivir durante los años de su retiro del mercado laboral con suficiente decoro, dignidad y comodidades que el sistema previsional del Estado no se lo permitiría nunca, lo que se hacía a través de mensajes publicitarios. Hoy, a más de 20 años del inicio de operaciones del sistema de las AFP, los trabajadores se encuentran con situaciones y condiciones de desinformación que al momento de su inscripción no fueron aclaradas por las AFP, lo cual constituye desinformación y práctica de publicidad engañosa. Hoy los trabajadores pueden advertir que todo lo que ofrecieron hace veintidós

Doctor en Ciencias Administrativas. Asesor y Consultor empresarial. Past Decano Nacional del Colegio de Licenciado en Administración del Perú, ha sido perito judicial de la Corte Superior de Justicia de Lima. Catedrático de pre y posgrado de diversas universidades del país, ex director académico del Instituto de Investigación de la FCA de la UNMSM. Premio en Investigación Científica 2014 en la UNMSM. 
años atrás no es verdad. Uno de los aspectos más críticos del establecimiento del Sistema Privado de Pensiones ha sido que la ley de creación del SSP fue dada dentro de un gobierno inconstitucional y dictatorial, con el cual se impuso el nuevo modelo de administración de los fondos de pensiones y el Estado deja de cumplir convenios y acuerdos internacionales sobre su compromiso de velar por la existencia y continuidad de un sistema previsional público. En virtud de esta realidad, el concepto y principio de la previsión social laboral del Estado no se cumple en millones de trabajadores en el Perú.

Palabras clave: Jubilación, Pensión, Previsión social

\begin{abstract}
The research aims to demonstrate that in the 90s in our country, time in which the ONP SNP was again in financial and economic peak time, so that the financial allocation for pension of pensioners receiving retirement at the time was far from being able to attend and cover not even $40 \%$ of basic food to survive. however in 1993 in a government dictatorial and unconstitutional court start is given by Decree Law No. 25897 to the new private pension system, system whereby the Administrators of Pension Funds, private entities created whose primeval business mission was to capture as much of retirees them leave the state system so that, on good account enroll and taper into a particular Administrator of Pension Funds, in order to transfer the registered together with a recognition bond in soles that It would be recognized by the ONP for their contributions made whenever these contributions reached 48 consecutive installments which may assess any of the periods established for the years 1992, 1996, 2001 for the processing of your bonus award, pecuniary amount the new member state grant to AFP in which he inscribed the affiliate, in order that it constitute a savings fund in their individual account as a contributor (Worker saver) in the new pension system. This was under the offer, the worker who saved more than two or three decades, the system had to offer through their advertising messages, a level of "pension" that would allow him to live during your retirement years labor market with sufficient decorum, dignity and comfort that the pension system is not state would never allow it. Today, more than twenty years of operation of the system AFPS, the workers are with plenty of situations and conditions of misinformation at the time of registration over twenty years ago were not given them by the AFPs, which is practice disinformation and misleading advertising in relation to non-compliance with the promises offered in the advertising campaign launch in some cases were joined without signing affiliate agreement. Today workers can see that, everything offered twenty-two years ago, is not true. One of the most critical of the establishment of the private pension system issues has been that the law establishing SSP was given in an unconstitutional and dictatorial government, with which the new model of management of pension funds and imposed State fails to meet international conventions and agreements on its commitment to ensuring the existence and status and continuity of a public pension system. Under this reality, the concept and principle of working welfare state is not fulfilled in million workers in Peru.
\end{abstract}

Keywords: Retirement, Pension, Welfare

\section{INTRODUCCIÓN}

El contraste entre la realidad económica y social en la que vive el trabajador jubilado peruano en el sistema privado de pensiones, comparado con el ofrecimiento de mejores niveles de vida mediante el futuro goce de una pensión digna, justa y equitativa que estos recibirían, hoy, a más de veinte años de aquel ofrecimiento, se puede ver que todo lo que le ofrecieron al trabajador jubilado fue un engaño. La campaña publicitaria de inicio de las operaciones de las AFP estuvo basada en la total desinformación, hecho adrede para persuadir y convencer de mejor forma a un afiliado poco advertido frente algo nuevo que se le presentaba como una mejor opción del sistema de la ONP. Los mensajes publicitarios que trasmitían las AFP en la televisión, radio, avisos en diarios, revistas y paneles no concuerdan con lo que los jubilados están recibiendo de ellas, lo cual constituye publicidad engañosa y estafa.

\section{El problema}

En junio de 1993, tiempo en el que las AFP inician sus operaciones comerciales de ofrecimiento de sus servicios de administrar los fondos de las pensiones de los trabajadores mediante un 
sistema de cuenta individual de ahorro, ofrecían sin mayores preguntas al posible afiliado que le tramitarían su bono de reconocimiento con lo cual aumentaría sus fondos en su cuenta individual de ahorros; de igual forma, le manifestaban que su sueldo sería aumentado y que eso se convertiría en un mayor beneficio para el trabajador.

Así mismo, la información que brindaban los promotores vendedores de las AFP y los mensajes del bombardeo publicitario en las radios, televisión, periódicos, revistas, y otros medios, le ofrecían al afiliado un nivel de pensión por jubilación muy superior a lo que recibirían en el SNP, incluso manifestaban que quienes permanecieran en dicho sistema corrían el riesgo de perder su jubilación por cuanto el sistema estaba quebrado, induciendo las decisiones de los afiliados con el uso de temor.

De otro lado, muchos empresarios negociaban en bloque la afiliación de todos sus trabajadores, para lo cual los presionaron para que firmaran su retiro del SNP y se afiliaran con la AFP que la empresa proponía. En caso el trabajador se negara a afiliarse lo amenazaban con despedirlo. Es obvio que las AFP se aprovecharon del nivel de desconocimiento y desinformación de los trabajadores por un lado, y de otro, el silencio y desinformación desde el Estado (Superintendencia de las Administradoras de Fondos de Pensiones Privados) en lo referente a qué condiciones deberían de cumplir los afiliados para poder gozar en el largo plazo de una pensión digna que les permitiera tranquilidad económica para ellos y sus familias, para lo cual se haría necesario la cantidad de años de aportación ininterrumpidos.

Otro de los aspectos que fueron aprovechados por las AFP fue la enorme estructura de informalidad laboral existente en el país. Es evidente que la estrategia de las AFP fue el captar la mayor cantidad de afiliados jóvenes ahorristas, eso les daría una liquidez mensual altamente importante para trabajarla y rendir cuentas a sus propietarios después de 20, 25 o 30 años después y de a poquitos, aún cuando el concepto es de ahorros de propiedad absoluta del ahorrista.

\section{OBJETIVOS}

\section{Objetivo específico}

- Resarcir beneficios que no le han sido entregados a los trabajadores ahorristas de Perú por el sistema privado de pensiones.
- Los aportantes al Sistema Nacional de Pensiones (SNP) tiene el goce de aguinaldos en fiestas patrias y navidad, beneficio negado al ahorrista del Sistema Privado de Pensiones.

- El monto de la devolución de los ahorros al trabajador no debe disminuir conforme pasan los años.

- Los ahorristas peruanos pagamos las comisionas más altas de Latino América a las AFP. El concepto con el que administran el dinero del trabajador es el de Cuenta Individual de Capitalización, dinero que captan por efecto de la imposición al trabajador de una ley coercitiva que debe cumplir la patronal y entregárselo a las AFP. Por estos ahorros coercitivos del trabajador las AFP no deben cobrar comisión. Es como si un banco nos cobrara comisiones por nuestros ahorros.

- El Sistema de las AFP vulnera los derechos constitucionales de la libre contratación y de la propiedad.

Los afiliados deben disponer libremente de sus ahorros para atender diversidad de necesidades que la vida les presenta en el camino a su jubilación.

\section{JUSTIFICACIÓN Y PROPUESTA}

La Universidad como fuente de producción de conocimiento y medio de solución de problemas sociales en el país debe buscar la verdad contenida en cualquier dimensión con problemática social como lo es el ahorro coercitivo y forzoso impuesto a los trabajadores peruanos mediante el Sistema Privado de Administración de Fondos privados de Pensiones (AFP), que a casi 25 años de su implementación, en la práctica no ha mejorado los montos de las pensiones de jubilación de los trabajadores, ni mucho menos estas devoluciones les alcanza para una vida con decoro, tranquila y digna; por el contrario, los ha empobrecido y complicado en la libre disponibilidad de su propiedad, siendo esto último un derecho constitucional que, en buena cuenta es ahorro, es una propiedad que pertenece al padre o madre de familia trabajador, en consecuencia el modelo ha afectado a la familia peruana al no poder disponer libremente de sus ahorros y adquirir los bienes y servicios que requiere para su prosperidad, desarrollo y bienestar, más aun si en el Perú nos jactamos de ser un país democrático y de estado de derecho en el qué, sus ciudadanos pueden disponer libremente de su propiedad en provecho propio. 
Sin embargo, la ley le impide su libre disponibilidad, incluso ante enfermedades y riesgos graves de salud familiar. Este modelo oligopólico de manejo del ahorro confiscador del dinero de los trabajadores es inquisitivo y esclavizante.

El modelo de Administración de los Fondos de Pensiones es un modelo coercitivo de la propiedad y voluntad del ser humano disfrazado de prevención social, que solo ha enriquecido al que más dinero tiene (a los propietarios del oligopolio, al sistema financiero y al Estado), y que ha empobrecido y continúa empobreciendo a los que menos tienen. Es en este sentido que la Universidad debe compartir la preocupación de los ciudadanos trabajadores y sus familias, a fin que estos recuperen la libertad de utilizar sus recursos económicos (ahorros) en la atención de cuanta vicisitud le presente la vida, y en las proyecciones de mejora continua, de desarrollo y prosperidad mediante la libre disponibilidad de sus propiedades en sus vidas. La Universidad es un estamento capaz de estudiar, investigar y analizar esta dimensión y actitud coercitiva y cómplice en el sometimiento y privación de la libertad de los ciudadanos y familias en cuanto al libre uso de sus bienes, a efectos que estos ahorros (recurso económicos) puedan utilizarlos en mayor generación de resultados económicos con efectos sinérgicos que propulsen aún más el desarrollo económico del país, y lo más importante, la utilización de los ahorros de los ciudadanos trabajadores y familias en la lucha contra la pobreza, mediante la inversión propia y directa de sus recursos.

En este sentido, la Universidad es un estamento capaz de efectuar propuestas de libertad. En cuanto a los beneficios para el país, es posible generar micro y pequeñas empresas mediante la asociatividad del conocimiento y experiencia aunado a aportes de capital entre personas jubiladas que estén interesadas en formular, evaluar y gestionar ellas mismas proyectos que generen mayores resultados económicos financieros que los escasos montos de devolución de ahorros mensuales que les devuelve la AFP.

\section{PROPUESTA}

- Disponibilidad mensual de sus ahorros para pago de gastos de educación de sus hijos, en el caso de desempleo.

- Permitir inmediatamente el uso del cien por ciento de sus ahorros al trabajador para la disminución y/o saneamiento de deudas bancarias hipotecarias. Esta inobservancia de la Ley $\mathrm{N} .^{\circ} 25897$ sobre la condición en la que se encuentran decenas de miles de ahorristas es empobrecedora, inconstitucional, $\mathrm{y}$ ha generado un enorme pasivo social que ha venido acrecentándose con los sucesivos gobiernos.

- Permitir la inmediata disponibilidad del cien por ciento de los ahorros del afiliado en casos de emergencia de la salud familiar en el primer grado de consanguinidad, y de $50 \%$ para casos de emergencia de salud familiar para los familiares del segundo grado de consanguinidad.

- Libre disponibilidad de los ahorros del afiliado y los de su cónyugue para la adquisición de un único bien inmueble para su habitación.

- Libre disponibilidad del cien por ciento de los ahorros del afiliado para su inversión en la creación de su propia empresa y trabajo.

El problema en sí consiste en qué los ofrecimientos del Sistema de Administración de Fondos de Pensiones Privados (AFP) en la década de los 90 a los futuros jubilados sobre la conveniencia de afiliarse al nuevo sistema de pensiones a efectos qué, en el largo plazo, éstos obtuvieran pensiones dignas que permitieran al jubilado y familia a vivir con suficiente tranquilidad económica, no se han cumplido. Hoy, los jubilados recuerdan y sienten con tristeza, el haber estado absolutamente desamparados en cuanto a recepción de información oficial que el Estado debió haberles brindado. Esta ausencia de información oficial generó las condiciones propicias para que las AFP, sus operadores y promotores los hayan sorprendido.

\section{HIPÓTESIS}

\section{Hipótesis general}

HG. El trabajador peruano, en condición de ahorrista según la Ley N. ${ }^{\circ} 25897$, cuenta con múltiples medios y formas para productivizar con mayores rendimientos sus ahorros, en relación a los escasos beneficios que obtienen en las AFP.

\section{Hipotesis específicas}

HE1. Los trabajadores ahorristas que se afiliaron a las AFP en la década de los 90, contaban con adecuada información oficial del Estado sobre como operaría el nuevo sistema de Administración de pensiones, y se encontraban debidamente informados por la publicidad brindada por las AFP de ese entonces. 


\section{META ESPECÍFICA}

Mostrar qué, la implantación del sistema previsional de Administración de Fondos de Pensiones Privado en nuestro país, no tuvo, tiene ni tendrá el móvil social laboral de mejorar la disponibilidad económica mensual del trabajador retirado de la actividad laboral en función a su avanzada edad. El amparo legal en el cual se basó y fundamentó su implementación está lleno de contradicciones, vicios y vacíos, fallas y contraposiciones legales que rebaten fácilmente su "legalidad".

Mostrar que el nivel de desinformación oficial desde el Estado que tuvieron los trabajadores en la década del 90 sobre el real funcionamiento del nuevo sistema de las AFP fue muy alto; bajo estas condiciones la gran mayoría de afiliados desconocía realmente de las bondades futuras que les brindaría realmente el nuevo sistema; sin embargo, la millonaria campaña publicitaria que aplicaron las AFP mediante el bombardeo publicitario los abrumó y actuaron de buena fe, y creyeron con la esperanza de un futuro mejor que hoy no ven ni viven.

Así mismo, la investigación pretende mostrar cuantitativamente el nivel de descontento y mortificación de los afiliados a las AFP en cuanto a los niveles de devolución mensual de su dinero (ahorros), que contradicen las condiciones de vida en la que realmente debe vivir un jubilado; vale decir qué, se debe cumplir lo que les ofreció la publicidad de las AFP en su campaña de lanzamiento en los años 90, en virtud a ello, el jubilado de hoy debería vivir dignamente pero eso no es así. Por el contrario, la devolución de los ahorros aportados por el trabajador está condicionada por las propias normas que las AFP se han dado al ser ellas quienes proveen a la Superintendencia de las AFP de información para que esta modifique y cambie las condiciones de devolución de los ahorros del trabajador, para implementar las normas y condiciones de operación que las AFP necesitan para su mejor operatividad y provecho propio, y poder operar de modo más eficiente; empero, esa eficiencia no es para trasladarla a los ahorristas sino para una mayor y mejor obtención de resultados económicos y financieros que ellas mismas planifican.

\section{CONTRIBUCIÓN E IMPACTO}

Se buscará sensibilizar al Estado a fin de que repare en la ilegalidad e inconstitucional que viene cometiendo desde hace 25 años con los trabajadores y familias del país al haber creado e implementado un Sistema de Administración de Fondo de Pensiones Privado con fines de proveer al sistema financiero nacional de recursos y fondos económicos para destinarlos a inversiones privadas nacionales y extranjeras, mediante la captación de "ahorro" forzado y coercitivo obtenido de los trabajadores y familias peruanas, con el propósito de convertir estos recursos captados en deuda a largo plazo, que será devuelta mediante un disfraz denominado "Pensión de jubilación del trabajador". La creación de éste sistema de captación de fondos ha dado origen a un enorme pasivo social, cuyos autores son múltiples.

Se buscará demostrar que la implantación de este sistema en el Perú se impuso dentro de un gobierno ilegítimo que defraudó y viene defraudando al trabajador peruano sin mayor escrúpulo, tan igual como en otros países donde se ha implementado como Chile. Sobre este país opinó el Premio Nobel de Economía, Joseph Stiglitz (2014), quien sostuvo que con lo ocurrido en el 2008 quedaron en evidencia varios mitos, como el relativo a lo recomendable que es que los fondos sean gestionados por privados.

Su bajada al sistema de pensiones fue lo que llamó el juego de suma cero, en que el sistema de pensiones privado puede invertir con alto riesgo en las bolsas internacionales y, si se produce una caída, es finalmente el Estado el que llega a cubrir los espacios dejados por la exposición de los fondos a estos riesgos. "A pesar de que toda la sociedad está pagando este enorme costo, aquí el que gana, ciertamente, es el sector financiero. Esto simplemente ha realzado el ingreso al sistema financiero en ese país, lo cual resulta en un juego de suma cero. Esto es una enorme pérdida para la sociedad y es muy importante alejarse del segundo pilar que se refiere al sistema privado", afirmó.

"Chile debiese estar sumamente preocupado por tener un sistema de pensiones privado, ya que es uno de los países más desiguales de la OCDE y ese modelo genera mayor desigualdad", planteó el economista estadounidense, agregando que Chile debiera tomar un camino que lo aleje "del segundo pilar del sistema privado". De hecho, recordó que en el mundo hay 23 países que privatizaron sus sistemas de pensiones, aunque ya siete de ellos revirtieron su decisión y habría varios más que lo están reconsiderando. 
En los años 90 la presencia de asesores e inversionistas chilenos entre otros extranjeros en nuestro país fue abrumadora, se puede decir que los chilenos estaban comprando el Perú. La presión de estos inversionistas para que en el Perú se implantase el modelo de las AFP a través de una sinergia oligopólica fue enorme; esto se evidencia en las fallas procesales de instrumentación jurídica para implantar el sistema de las AFP.

Un relato muy interesante sobre esta situación es el que plantea Cisneros (2014), quien en un artículo comenta lo siguiente: “... el Estado peruano se ha coludido con la empresa privada en el mayor robo institucionalizado contra el ciudadano, el asalto a plazos mejor marketeado de nuestra historia... El actual sistema peruano de AFP es confiscatorio, inconstitucional, excluyente y diseñado para beneficiar principalmente a la empresa en desmedro del ciudadano.... Aquí el Marketing de las AFP no te dice que el sistema está diseñado para que no puedas acceder en vida al íntegro de tu dinero; la Superintendencia de Banca y Seguros (SBS) argumenta que es un sistema pensado para la familia del aportante".

\section{METODOLOGÍA}

Se ha efectuado una investigación exploratoria histórico-descriptiva sobre publicaciones efectuadas en la década del 90 por las AFP y el Estado. La revisión ha sido efectuada en medios gráficos, videos, páginas web, Internet, revistas y secuencia cronológica de dación de decretos y leyes con los cuales se validó la implantación del Sistema de Administración de Fondo de Pensiones Privado. De igual forma, en la investigación se han utilizado metodologías cuantitativas a efectos de medir la opinión de los jubilados sobre su situación económica en la que se encuentran, luego de haber ahorrado para su vejez durante $20,25,30$ y hasta 45 años en aportes tanto al SNP como a las AFP.

\section{POBLACIÓN}

Para medir la opinión de los jubilados en la gran Lima sobre el nivel de satisfacción en cuanto a la conformidad y cumplimiento sobre lo que les ofreció el Sistema Privado de Pensiones en la década de los 90 para su afiliación y lo que realmente reciben hoy, se aplicaron estudios cuantitativos muéstrales aleatorios a 400 jubilados de Lima durante los meses de junio y julio del 2015.

\section{MUESTRA}

La muestra se determinó dentro de los parámetros siguientes:

Caso más desfavorable $\mathrm{P}=\mathrm{Q}$

Desviación estándar $(\sigma)=2$

Error estimado $=(0.5)$

Cantidad de encuestas $=\mathrm{n}$

\section{FÓRMULA}

$$
\begin{aligned}
& \mathrm{n}=\sigma 2\left[(\mathrm{P})^{*}(\mathrm{Q})\right] / \text { e2 }=400 \text { encuestas } \\
& \text { TRABAJO DE CAMPO }
\end{aligned}
$$

Las entrevistas se efectuaron a personas jubiladas indistintamente de su género, que reciben devolución de sus ahorros por una AFP bajo la connotación de "pensión de jubilación". La selección y aplicación de la unidad representativa de información fue totalmente aleatoria.

Para la realización de la encuesta en el trabajo de campo intervino un equipo de estudiantes de la Facultad de Ciencias Administrativas conformado por 60 alumnos debidamente instruidos en el manejo del cuestionario aplicado. Posteriormente la información sobre las personas que fueron entrevistadas fue filtrada y contrastada con los datos de la Superintendencia de las AFP. Este trabajo de campo se efectuó durante los meses de junio y julio del 2015.

\section{RESULTADOS}

Los resultados se obtuvieron mediante las tabulaciones, cálculos y análisis efectuados al total de la muestra de 400 encuestas efectuadas a jubilados, mediante la aplicación de un cuestionario diseñado sobre la base de las variables en estudio. El cuestionario fue probado en un piloto de prueba de 30 encuestas antes de su aplicación en la muestra, y satisfizo en cuanto a la aplicabilidad, funcionabilidad y medición de las variables.

\section{Resultados cuánticos}

Se le preguntó al encuestado cuál había sido el monto de sus últimos cinco sueldos con los que se jubiló. El $52 \%$ de los jubilados encuestados manifestaron haber tenido sueldos inferiores a S/. 1,500. De igual forma, el 15\% manifestó que su sueldo fue inferior a S/. 2,000; mientras que el $10 \%$ manifestó S/. 2,500; y el 6\% que recibía un sueldo inferior a $\mathrm{S} / .3,000$. 
Como podemos apreciar un alto porcentaje de $52 \%$ recibía sueldos inferiores a S/. 1,500. De igual forma se observa que un alto porcentaje $(83 \%)$ recibía menos de $\mathrm{S} / .3,000$, lo cual muestra que los sueldos en nuestro país son excesivamente bajos.

Cuadro N. ${ }^{\circ}$ 1. Rango de sus últimos cinco sueldos

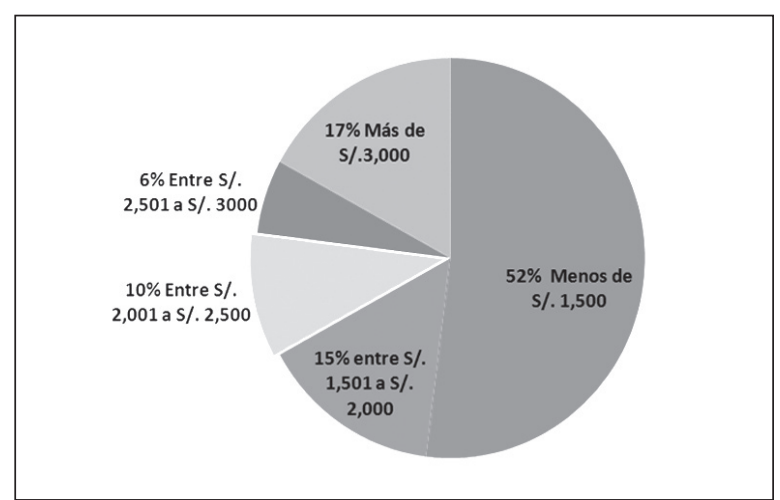

Fuente: Elaboración propia.

Sobre la pregunta efectuada a los jubilados sobre su pertenencia al SNP u ONP, el $80 \%$ respondió haber pertenecido a la ONP, lo cual significa una gran captación de trabajadores aportantes desertores de la ONP que tuvieron las AFP. Tan solo un $20 \%$ de sus afiliados iniciaron como aportantes en el nuevo sistema.

Cuadro N. ${ }^{\circ}$ 2. ¿Perteneció usted al Sistema Nacional de Pensiones?

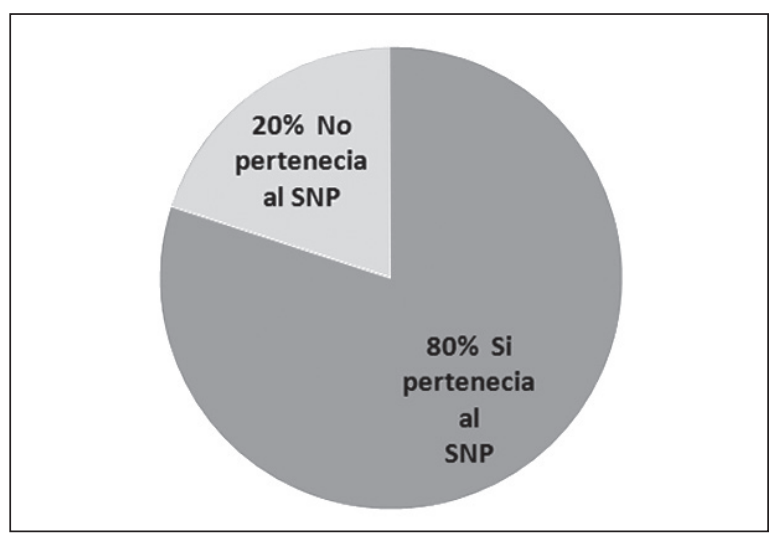

Fuente: Elaboración propia.

Parte de la gran campaña publicitaria de las AFP en la década de los 90 fue el ofrecimiento de que ellas mismas se encargarían de la tramitación del bono de reconocimiento que otorgaría el Estado a los que se afiliaran en el nuevo sistema de pensiones. De los encuestados, tan solo el 55\% ha obtenido bono de reconocimiento, mientras que un $45 \%$ se encuentra totalmente desatendido y descontento por este incumplimiento.
Cuadro N. ${ }^{03}$. ¿Recibió su bono de reconocimiento?

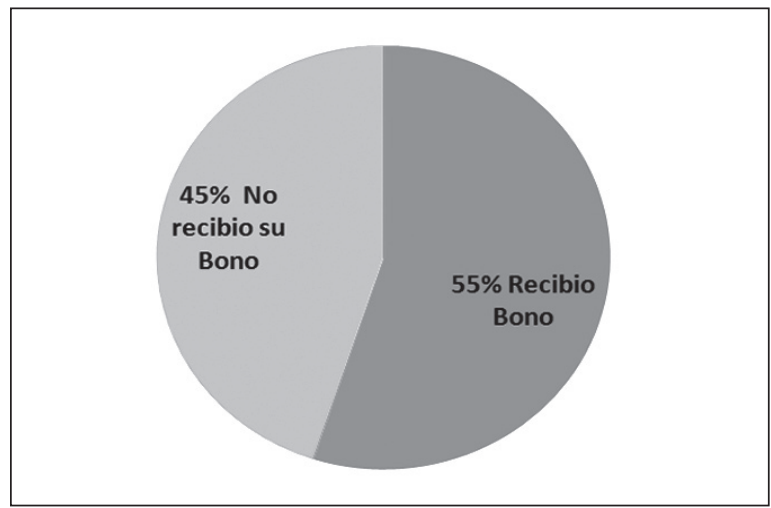

Fuente: Elaboración propia.

Sobre la pregunta a los jubilados de si consideraban justo el monto de devolución que recibían por sus ahorros más el rendimiento de estos durante todos los años que habían ahorrado en la AFP, el 82\% lo consideró injusto, el $2 \%$ no supo dar opinión al respecto, y el $16 \%$ lo consideró justo.

Cuadro N. ${ }^{\circ} 4$. ¿Considera justo o injusto lo devuelto mensualmente por las AFP?

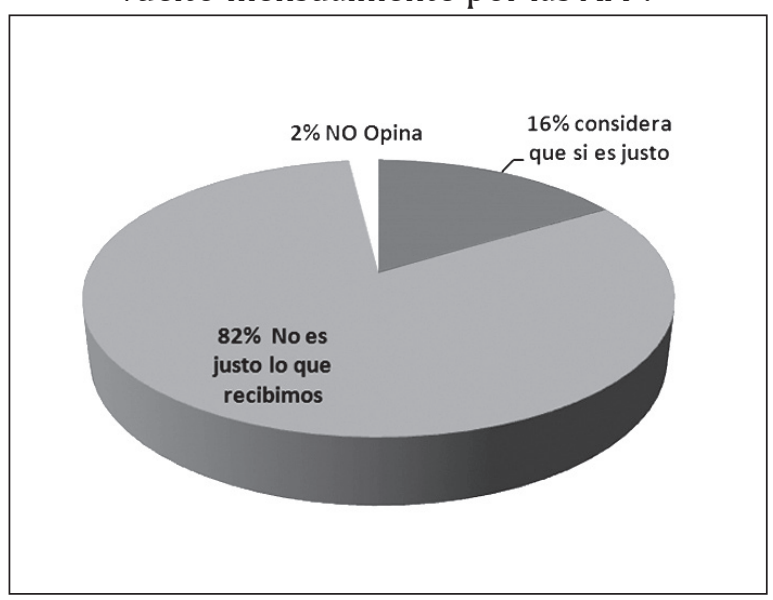

Fuente: Elaboración propia.

La pregunta efectuada a los jubilados sobre si la publicidad y las maravillas que anunciaban en el contenido de sus mensajes publicitarios efectuados por las AFP en su campaña de lanzamiento en 1993 habían influenciado en ellos, imaginando que verdaderamente recibirían en el futuro pensiones dignas que les permitiera vivir tranquilos económicamente a ellos y sus familias, el 70\% de los jubilados manifestó que la publicidad y el trato amable y generoso en cuanto a obsequios que entregaban los promotores y vendedores los habían persuadido; empero, hoy sienten que todo fue un verdadero engaño. 
Cuadro No5. ¿La publicidad influenció en su decisión de afiliarse?

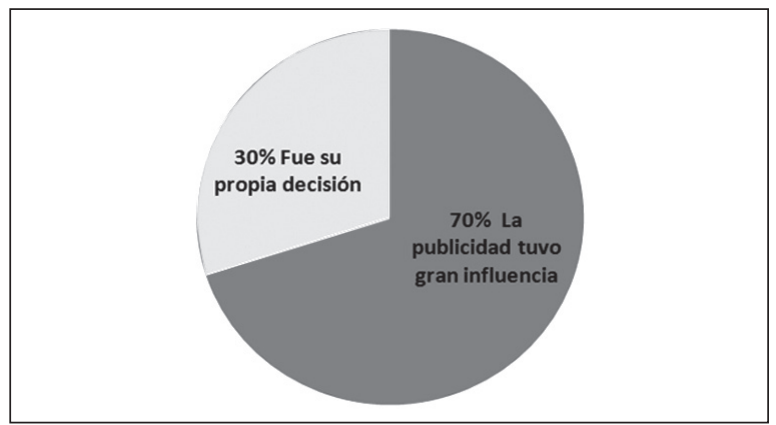

Fuente: Elaboración propia.

\section{DISCUSIÓN}

Los resultados de los análisis cualitativos y cuantitativos de nuestra investigación demuestran que el diseño del Sistema de Administración de Fondos Privados de Pensiones (AFP) no ha satisfecho las expectativas que éstas generaron a través de los mensajes publicitarios con los que bombardearon a los ciudadanos trabajadores y familias peruanas en su enorme y abrumadora campaña publicitaria de lanzamiento en 1993.

El Estado peruano de aquella época pecó de modo cómplice, manteniendo un silencio de desinformación oficial a los trabajadores y sus familias sobre cómo operaria el nuevo Sistema Privado de Pensiones. Sin embargo, el análisis histórico sobre la dación e instrumentación jurídica de leyes, creación de la Superintendencia de Administración de los Fondos Privados de Pensiones, reglamentos y normas se dieron con tanta celeridad que abren una sospecha razonable sobre el enorme interés de los agentes participantes en este nuevo sistema, especialmente los grupos chilenos y su experiencia en su país.

Hoy en el 2015, a 23 años de la implementación de éste sistema en el Perú, el malestar generado en la población laboral y familiar es muy significativo y notorio por el incumplimiento de todo lo ofrecido en la década del 90 . En la actualidad, las devoluciones de los ahorros y las ganancias producidas por estos no le permiten al jubilado gozar de aquella vida futura que le ofrecieron de vivir con dignidad y tranquilidad económica, todo ello fue una farsa que los engañó mediante la publicidad utilizada en la campaña de la década del 90. Se aprovechó de la ausencia de información oficial del Estado sobre los efectos negativos en el monto de las pensiones que tendría este nuevo sistema de jubilación, en una dimensión laboral con un altísimo nivel de informalidad.

\section{CONCLUSIÓN}

1. La hipótesis general de la investigación fue demostrada mediante análisis comparativos de rendimientos de un capital de S/. 5,000 que por actividad propia del trabajador como empresa unipersonal, y trabajando en su propio domicilio, con una rotación de capital de $100 \%$ una vez al mes, con un margen comercial de $30 \%$, supera ampliamente al rendimiento promedio anual capitalizable del $9 \%$ ofrecido por una AFP.

2. La hipótesis específica de la investigación se desechó al ser contrastada con la hipótesis Nula (Ho). Los trabajadores ahorristas no se encontraban informados oficialmente por el Estado sobre cómo operaria el Sistema de las AFP y la información publicitaria no era real, incumpliéndose lo que ofrecían a largo plazo. Los valores de Chi cuadrado fueron contrapuestos a los estadísticos de prueba y se aceptó la hipótesis nula.

3. El análisis histórico de la promulgación de las leyes, normas y disposiciones que se dieron para la creación de éste nuevo sistema privado de jubilación dentro de un gobierno de facto e ilegitimo en la década de los 90 , demuestran que existió celeridad y precipitación nunca antes vista en el ordenamiento jurídico del país para la promulgación de una ley.

4. El análisis histórico de los mensajes y material publicitario que utilizaron las AFP en su campaña de lanzamiento en la década del 90 muestra que utilizaron publicidad confusa y engañosa a los trabajadores. El Estado tuvo un comportamiento cómplice con el nivel de desinformación para los trabajadores, prácticamente estos se sintieron abandonados con respecto a esta necesidad de información necesaria para la toma de decisiones.

5. A 23 años del imperio de la Ley $\mathrm{N}^{\circ} 27898$, un alto porcentaje del orden del $82 \%$ de los jubilados de la gran Lima considera que el Sistema Privado de Administración de Fondo de Pensiones es injusto. El 70\% de los jubilados manifestaron que la publicidad que utilizaron las AFP en la década de los 90 influenció fuertemente en su decisión de afiliarse, aun cuando ellos se sentían totalmente desinformados oficialmente por el Estado, que no cumplió con tan importante función. 
6. Un $45 \%$ de los jubilados no gozan del bono de reconocimiento que ofreció la ONP en la década de los años 90, en virtud que ésta no informó durante la campaña publicitaria de lanzamiento de las AFP sobre quién tendrían derecho al bono de reconocimiento. De igual forma las AFP no cumplieron con el ofrecimiento de tramitar el bono de reconocimiento del afiliado.

7. Más del $52 \%$ de los jubilados manifestaron que el monto de devolución de sus ahorros, pensión de jubilación que reciben de la AFP a la cual pertenecen es insuficiente, no les alcanza y es muy precario y lejano para cubrir y atender sus necesidades básicas y las de su familia, no pueden decir que viven dignamente y en tranquilidad económica.

8. Del análisis de estas variables se colige que las AFP no son instituciones con gestión eficiente de los ahorros de los trabajadores que hayan podido demostrar que efectivamente han contribuido al mejoramiento de las pensiones de jubilación de los trabajadores. Por el contrario, han servido para demostrar que el valor del monto de la devolución mensual de sus ahorros los empobrece a él y a su familia.

\section{REFERENCIAS BIBLIOGRÁFICAS}

LA REPÚBLICA (2012). AFP: CONTINÚAN LAS CRÍTICAS A LA REFORMA DEL SISTEMA
PRIVADO DE PENSIONES. Edición impresa el 10 de julio de 2012. Lima - Perú.

CISNEROS MENDEZ, CLAUDIA (2014). AFP: EL MAYOR DESPOJO INSTITUCIONALIZADO II. DIARIO OPINIÓN. 31 DE AGOSTO DEL 2014 LIMA - PERÚ.

ALFARO, GABRIELA (2014). LOS FONDOS DE PENSIONES EN EL PERÚ. 2015 Prezi Inc. Condiciones.

MOHME SEMINARIO, GUSTAVO (2014). EDITORIAL DEL DIARIO LA REPUBLICA. "UNA LEY RESPONSABLE" AGOSTO 2014. PERÚ.

GARCIA BELAUNDE, VÍCTOR A (2014). ARTÍCULO "LOS QUE ATACAN A WEB ESTÁ, FINANCIADOS POR LAS AFP" DIARIO. LA REPÚBLICA. AGOSTO 2014. PERÚ.

EL COMERCIO (2015). LAS AFP, POR RICHARD WEBB. Edición del 04 de agosto del 2014. Lima -Perú

PERU 21. BAJA AFILIACIÓN EN LAS AFP, Y AUMENTA PREFERENCIA POR LA ONP. Edición del 13 de Septiembre del 2013.

STIGLITZ, JOSEPH (2014). PREMIO NOBEL DE ECONOMÍA 2001. NO + AFP. Diario el pulso - CHILE.

SÁNCHEZ, ELIO (2014). HOMUS ECONOMICUS EGOÍSTA E INDIVIDUAL. HTTP://GOO.GL/ GSY3RC - LIMA - PERÚ. 